\title{
Paquete ZOIP de R para analizar datos proporcionales inflados con ceros o unos
}

\section{ZOIP package in $\mathbf{R}$ to analyse inflated proportional data with zeros and/or ones}

Juan Camilo Díaz Zapata jucdiazza@unal.edu.co
Freddy Hernández Barajas b

fhernanb@unal.edu.co

Olga Cecilia Usuga Manco ${ }^{c}$

olga.usuga@udea.edu.co

\begin{abstract}
Resumen
Los datos obtenidos a partir de variables medidas como porcentajes, tasas y proporciones, son llamados datos proporcionales y se encuentran ubicados por lo general en el intervalo $(0,1)$. Diferentes distribuciones se han desarrollado para caracterizar este tipo de variables, sin embargo, existe la posibilidad de que este tipo de variables puedan dar resultados de cero o uno. Haciendo que autores como Ospina \& Ferrari (2012) y Rigby \& Stasinopoulos (2005) implementaran una distribución Beta inflada con ceros unos, diferenciadas solo por su parametrización. Otros autores como Galvis \& Lachos (2014) han trabajo con otras distribuciones para datos proporcionales inflados, como la distribución simplex, sin embargo, no se ha encontrado una distribución que reúna las principales distribuciones para datos proporcionales inflados con ceros unos. En este trabajo se presenta el paquete ZOIP del sistema de computación $\mathrm{R}$ para la implementación de la distribución ZOIP (Zeros Ones Inflated Proportional) que reúne la distribución simplex y beta bajo diferentes parametrizaciones y estima los parámetros de dicha distribución. La estimación de los parámetros de la distribución ZOIP se lleva acabo vía máxima verosimilitud. Se realizaron estudios de simulación que muestran la convergencia satisfactoria de los parámetros y se presenta el ajuste de una distribución ZOIP a datos reales.
\end{abstract}

Palabras clave: datos proporcionales, distribución ZOIP, máxima verosimilitud, R.

\footnotetext{
a Estudiante de maestría en estadística, Universidad Nacional de Colombia, Sede Medellín.

bProfesor asistente, Universidad Nacional de Colombia, Sede Medellín.

${ }^{\mathrm{c}}$ Profesora asociada, Universidad de Antioquia, Medellín.
} 
Data obtained from variables measured as percentages, rates and proportions are called proportional data and these variables are usually within the $(0,1)$ interval. Although, different distributions have been developed to characterize these variables, it is possible that some variables take the extreme values at zero and one. Some researchers as Ospina \& Ferrari (2012) and Rigby \& Stasinopoulos (2005) proposed the inflated Beta distribution with zeros and/or ones, differentiated only by their parameterization. Other authors such as Galvis \& Lachos (2014) have worked with inflated proportional data, considering other distributions such as simplex distributions. Nevertheless, it has not been found any distribution which combines the main characteristic of several distributions for solving these issue. Thus, this paper presents the ZOIP package (Zeros Ones Inflated Proportional), developed on the computation system $\mathrm{R}$, this package combines both Beta and simplex distribution and estimates its parameters given the parameters for the original distributions. The parameters estimation process, is performed by maximum likelihood method. Finally, simulations studies have been performed which show the accuracy of the parameters convergence and the adjustment of a ZOIP distribution on real data.

Keywords: Proportional data, ZOIP Distribution, Maximum likelihood, R.

\section{Introducción}

En modelación estadística es posible encontrarnos con variables respuesta como proporciones, porcentajes o tasas que se encuentran en el intevalo $(0,1)$. La distribución más utilizada en la literatura para caracterizar este tipo de variables es la distribución beta con soporte en el intervalo $(0,1)$, la cual ha sido reparametrizada por autores como Ferrari \& Cribari-Neto (2004) y Rigby \& Stasinopoulos (2005); otras distribuciones no tan comunes en la literatura, pero que caracterizan este tipo de variables son la distribución simplex (Jørgensen 1997), beta-rectangular (Hahn 2008) y la distribución LogitSep (Hossain 2015). Por otra parte, es común que los porcentajes o las proporciones puedan dar valores iguales a cero o uno, representando la ausencia o presencia total de la característica de interés, respectivamente; las distribuciones descritas anteriormente no pueden ser admisibles para este tipo de variables, es por esto que se han desarrollado distribuciones infladas con ceros y unos, para tratar estos casos, como lo hizo Ospina \& Ferrari (2010), quienes presentan una distribución beta inflada en la que hacen una combinación entre una distribución discreta para la parte de los valores que pueden tomar cero o uno y una parte continua para los valores continuos entre cero y uno. Rigby \& Stasinopoulos (2005) incluyen dentro de sus modelos gamlss la distribución beta inflada con ceros y unos según su parametrización.

Esto ha dado pie para que diferentes autores hayan empezado a desarrollar diferentes modelos de regresión para tratar este tipo variables, Ospina \& Ferrari (2012) propusieron una clase general de modelos de regresión beta inflados con cero o 
uno, además Kosmidis et al. (2015) han estudiado dichos modelos inflados recientemente, pero con una distribución distinta a la presentada por Ospina \& Ferrari (2012). Galvis \& Lachos (2014) presentan modelos de regresión de diferentes distribuciones de datos proporcionales inflados con ceros unos mediante metodologías de estimación bayesianas.

Muchos autores han implementado distribuciones para datos proporcionales en el sofware estadístico R, Zeileis et al. (2010) implementan el paquete betareg donde se encuentran los modelos de regresión Beta propuestos por Ferrari \& CribariNeto (2004). Qiu et al. (2008) implementan el paquete simplexreg para realizar análisis de distribución y regresión sobre una distribución simplex, para datos proporcionales no inflados. Cepeda-Cuervo et al. (2016) presentan la implementación de modelos de regresión beta bajo un enfoque bayesiano utilizando el paquete Bayesianbetareg. Otros autores como Rigby \& Stasinopoulos (2008) incluyen en el paquete gamlss la distribución beta inflada con ceros unos, además de, la posibilidad de realizar modelos de regresión sobre ellos.

Aunque muchos autores han implementado las distribuciones para datos proporcionales inflados con ceros y unos, ninguno ha presentado una propuesta como la de reunir en una sola distribución las diferentes distribuciones para datos proporcionales y sus diferentes parametrizaciones, además de implementarla en un solo paquete, como se presenta en el paquete ZOIP en R Core Team (2017).

El artículo se encuentra organizado de la siguiente manera: primero se presentan las distribuciones más representativas para datos proporcionales, en la sección 3 se presenta la distribución para datos proporcionales inflados con ceros y/o unos ZOIP (Zeros Ones Inflated Proportional), seguido por el desarrollo analítico de la estimación de los parámetros de la distribución ZOIP vía máxima verosimilitud, en la sección 5 se aplica el ajuste de una distribución ZOIP en un estudio de simulación y para datos reales, por último se presenta el paquete ZOIP.

\section{Distribución para datos proporcionales}

Por lo general en los casos de modelación donde la variable de interés es una proporción, un porcentaje o una tasa, no se suelen ser analizadas con la distribución normal, debido a que el soporte de la normal es la recta real $\mathbb{R}$, además en este tipo de variables es común la asimetría e incluso la bimodalidad; por esta razón, en la literatura estadística se han propuesto distribuciones para este tipo de comportamientos, como la distribución beta, que cuenta con diferentes parametrizaciones (Ferrari \& Cribari-Neto (2004) y Rigby \& Stasinopoulos (2005)) y la distribución simplex propuesta por Jørgensen \& Barndorff-Nielsen (1991). De igual manera, otras distribuciones más particulares como la beta-rectangular (Hahn 2008) y LogitSep (Hossain 2015) se acoplan a este comportamiento. A con- 
tinuación se presentan las funciones de densidad de probabilidad, la media, la varianza y dependencias de algunas de las distribuciones mencionadas.

\subsection{Distribución beta}

Si una variable aleatoria $Y$ definida entre cero y uno, tiene distribución beta con parámetros $p$ y $q$ se acostumbra a denotarla por $Y \sim \operatorname{Be}(p, q)$ y la función de densidad de probabilidad de la distribución es dada por:

$$
f(y ; p, q)=\frac{\Gamma(p+q)}{\Gamma(p) \Gamma(q)} y^{p-1}(1-y)^{q-1},
$$

Donde los parámetros $p>0, q>0$ y $\Gamma(\cdot)$ es la función gamma. El valor esperado y la varianza de $Y$ están dadas por:

$$
\begin{aligned}
E(Y) & =\frac{p}{p+q} \\
\operatorname{Var}(Y) & =\frac{p q}{(p+q)^{2}(p+q+1)}
\end{aligned}
$$

\subsection{Distribución beta parametrización Ferrari y Cribari-Neto (2004)}

Ferrari \& Cribari-Neto (2004) propusieron otra parametrización para la distribución beta en función de los parámetos $\mu$ y $\phi$, donde $\mu$ corresponde a la media de la distribución y $\phi$ es interpretado como un parámetro de precisión. Si $0<Y<1$ y $Y \sim \operatorname{Be}(\mu, \phi)$ la función de densidad de probabilidad de la distribución está dada por:

$$
f(y ; \mu, \phi)=\frac{\Gamma(\phi)}{\Gamma(\mu \phi) \Gamma((1-\mu) \phi)} y^{\mu \phi-1}(1-y)^{(1-\mu) \phi-1}
$$

Donde $0<\mu<1$ y $\phi>0$. El valor esperado y la varianza de $Y$ están dados por:

$$
\begin{aligned}
E(Y) & =\mu \\
\operatorname{Var}(Y) & =\frac{\mu(1-\mu)}{1+\phi}
\end{aligned}
$$

Además, note que la parametrizacion de la distribución beta es equivalente a la de Ferrari \& Cribari-Neto (2004) cuando:

$$
\begin{aligned}
& p=\mu \phi \\
& q=(1-\mu) \phi
\end{aligned}
$$




\subsection{Distribución beta parametrización Rigby y Stasinopou- $\operatorname{los}(2005)$}

Rigby \& Stasinopoulos (2005) propusieron una nueva parametrización para la distribución beta con parámetros $\mu$ y $\sigma$, donde $\mu$ es la media de la distribución y $\sigma$ es interpretado como un parámetro de dispersión, se dice que $Y \sim \operatorname{Be}(\mu, \sigma)$ con $0<Y<1$, si la función de densidad de probabilidad de la distribución está dada por:

$$
f(y ; \mu, \sigma)=B(\mu, \sigma) y^{\mu\left(\left(1-\sigma^{2}\right) / \sigma^{2}\right)-1}(1-y)^{(1-\mu)\left(\left(1-\sigma^{2}\right) / \sigma^{2}\right)-1}
$$

Donde $B(\mu, \sigma)=\frac{\Gamma\left(\left(1-\sigma^{2}\right) / \sigma^{2}\right)}{\Gamma\left(\mu\left(\left(1-\sigma^{2}\right) / \sigma^{2}\right)\right) \Gamma\left((1-\mu)\left(\left(1-\sigma^{2}\right) / \sigma^{2}\right)\right)}$,

Donde $0<\mu<1$ y $0<\sigma<1$. La media y la varianza de $Y$ están dadas por:

$$
\begin{aligned}
E(Y) & =\mu \\
\operatorname{Var}(Y) & =\sigma^{2} \mu(1-\mu)
\end{aligned}
$$

Además note que la parametrizacion de la distribucion beta es equivalente a la de Rigby \& Stasinopoulos (2005) cuando:

$$
\begin{aligned}
& p=\frac{\mu\left(1-\sigma^{2}\right)}{\sigma^{2}} \\
& q=\frac{(1-\mu)\left(1-\sigma^{2}\right)}{\sigma^{2}}
\end{aligned}
$$

\subsection{Distribución simplex}

La distribución simplex fue introducida por Jørgensen \& Barndorff-Nielsen (1991) y es un caso particular de los modelos de dispersión propuestos por Jørgensen (1997), dicha distribución depende de los parámetros $\mu$ que corresponde a la media de la distribución y $\sigma^{2}$, la cual corresponde a un parámetro de dispersión. Si $0<Y<1$ y $Y \sim S^{-}\left(\mu, \sigma^{2}\right)$ la función de densidad de probabilidad esta dada por:

$$
f\left(y ; \mu, \sigma^{2}\right)=\left\{2 \pi \sigma^{2}[y(1-y)]^{3}\right\}^{-1 / 2} \exp \left\{-\frac{y(1-y) \mu^{2}(1-\mu)^{2}}{2 \sigma^{2}(y-\mu)^{2}}\right\}
$$

Donde $0<\mu<1$ y $\sigma>0$. Además el valor esperado y la varianza están dadas por: 


$$
\begin{aligned}
E(Y) & =\mu \\
\tau^{2} & =\mu(1-\mu)-\frac{1}{\sqrt{2 \sigma^{2}}} \exp \left\{\frac{1}{2 \sigma^{2} \mu^{2}(1-\mu)^{2}}\right\} \Gamma\left\{\frac{1}{2}, \frac{1}{2 \sigma^{2} \mu^{2}(1-\mu)^{2}}\right\} \\
\operatorname{Var}(Y) & =1 / \tau^{2}
\end{aligned}
$$

Donde $\Gamma(a, b)$ está dado por la función $\Gamma$ incompleta definido como $\Gamma(a, b)=$ $\int_{b}^{\infty} t^{a-1} b^{t} d t$, ver más en Zhang et al. (2016).

\section{Distribución ZOIP (Zeros Ones Inflated Pro- porcional)}

En las distribuciones vistas en la sección 2 se evidenciaron algunas distribuciones que se ajustan al comportamiento de datos proporcionales, porcentajes o tasas que están en el intervalo $(0,1)$; sin embargo, es común que estos datos tomen valores en cero y uno que representarían la ausencia o presencia total de cierta característica, por lo que no sería posible ajustar los datos a las distribuciones vistas anteriormente y es por eso que en este trabajo se propone la distribución ZOIP, como un conjunto de distribuciones para datos proporcionales inflados con ceros y unos.

La distribución para datos proporcionales inflados con ceros y unos se compone de la mezcla de tres distribuciones, dos de ellas discretas, que son distribuciones degeneradas en cero y uno, y una tercera distribución continua que además es una función de densidad de probabilidad para datos proporcionales, como las presentadas anteriormente.

Si la variable aleatoria $Y$ tiene distribución ZOIP con parámetros $\mu, \sigma, p_{0}$ y $p_{1}$, se denotará como $Y \sim \operatorname{ZOIP}\left(\mu, \sigma, p_{0}, p_{1}\right)$, la función de densidad de probabilidad está dado por:

$$
g\left(y ; \mu, \sigma, p_{0}, p_{1}\right)= \begin{cases}p_{0} & \text { si } y=0, \\ p_{1} & \text { si } y=1, \\ \left(1-p_{0}-p_{1}\right) f(y ; \mu, \sigma) & \text { si } y \in(0,1)\end{cases}
$$

Donde $p_{0} \geq 0$ y $p_{1} \geq 0$ representan la probabilidad de que la variable aleatoria $Y$ sea igual a cero y uno, respectivamente, además $0 \leq p_{0}+p_{1} \leq 1$ y $f(y ; \mu, \sigma)$ representa alguna de las funciones de densidad de probabilidad para datos proporcionales, descritas en la sección anterior. La media y varianza de $y$, están dadas por

$$
E(Y)=p_{1}+\left(1-p_{0}-p_{1}\right) E^{*}(Y)
$$




$$
\begin{aligned}
\operatorname{Var}(Y)=p_{1} & (1-p 1)+ \\
& +\left(1-p_{0}-p_{1}\right)\left[\operatorname{Var}^{*}(Y)+\left(p_{0}+p_{1}\right)\left[E^{*}(Y)\right]^{2}-2 E^{*}(Y) p_{1}\right]
\end{aligned}
$$

Donde $E^{*}(Y)$ y $\operatorname{Var}^{*}(Y)$ denotan la esperanza y varianza asociados a la distribución $f(y ; \mu, \sigma)$.

Tabla 1: Rol que toman los parámetros de la distribución ZOIP de acuerdo a la distribución y parametrización elegida.

\begin{tabular}{|c|l|c|}
\hline Parámetro & Distribución/Parametrización & Rol en la distribución ZOIP \\
\hline \multirow{5}{*}{$\mu$} & ZOIP-Beta R-S & $\mu$ \\
& ZOIP-Beta F-C & $\mu$ \\
& ZOIP-Beta & $p$ \\
& ZOIP-simplex & $\mu$ \\
\hline \multirow{5}{*}{$\sigma$} & ZOIP-Beta R-S & $\sigma$ \\
& ZOIP-Beta F-C & $\phi$ \\
& ZOIP-Beta & $q$ \\
& ZOIP-simplex & $\sigma$ \\
\hline \multirow{5}{*}{$p_{0}$} & ZOIP-Beta R-S & $p_{0}$ \\
& ZOIP-Beta F-C & $p_{0}$ \\
& ZOIP-Beta & $p_{0}$ \\
& ZOIP-simplex & $p_{0}$ \\
\hline \multirow{5}{*}{$p_{1}$} & ZOIP-Beta R-S & $p_{1}$ \\
& ZOIP-Beta F-C & $p_{1}$ \\
& ZOIP-Beta & $p_{1}$ \\
& ZOIP-simplex & $p_{1}$ \\
\hline
\end{tabular}

En este artículo se plantea la distribución ZOIP-Beta bajo tres diferentes parametrizaciones, esto para que el usuario elija la parametrización más conveniente de acuerdo con su caso de estudio. En la tabla 1 se muestra el rol que toman los parámetros de $\mu$ y $\sigma$ siguiendo la elección de la parametrización de la distribución ZOIP-beta, de este modo, si la distribución ZOIP-beta $\left(\mu, \sigma, p_{0}, p_{1}\right)$ es con parametrización Ferrari \& Cribari-Neto (2004), entonces el parámetro $\sigma$ tomará el rol del parámetro $\phi$ de la distribución y si la parametrización es ZOIP-beta $\mu$ y $\sigma$ tomarán el rol de $p$ y $q$ respectivamente. En las demás distribuciones y parametrizaciones $\mu$ y $\sigma$ tomarán los valores y dominios correspondientes a su distribución.

La distribución ZOIP se encuentra inflada con ceros y unos, es decir, bilateralmente, pero existe la posibilidad que hayan casos de estudio en que se encuentren datos inflados con unos únicamente, por lo que $p_{0}=0$ y por lo tanto se estará llamando no una distribución ZOIP, sino una distribución OIP (Ones Inflated Proporcional) y si los datos se encuentran inflados con ceros únicamente, es decir $p_{1}=0$ se tendrá una distribución ZIP (Zeros Inflated Proporcional), si los datos no se encuentran inflados, entonces $p_{0}=p_{1}=0$ y la distribución ZOIP será una distribución para datos proporcionales clásica. 

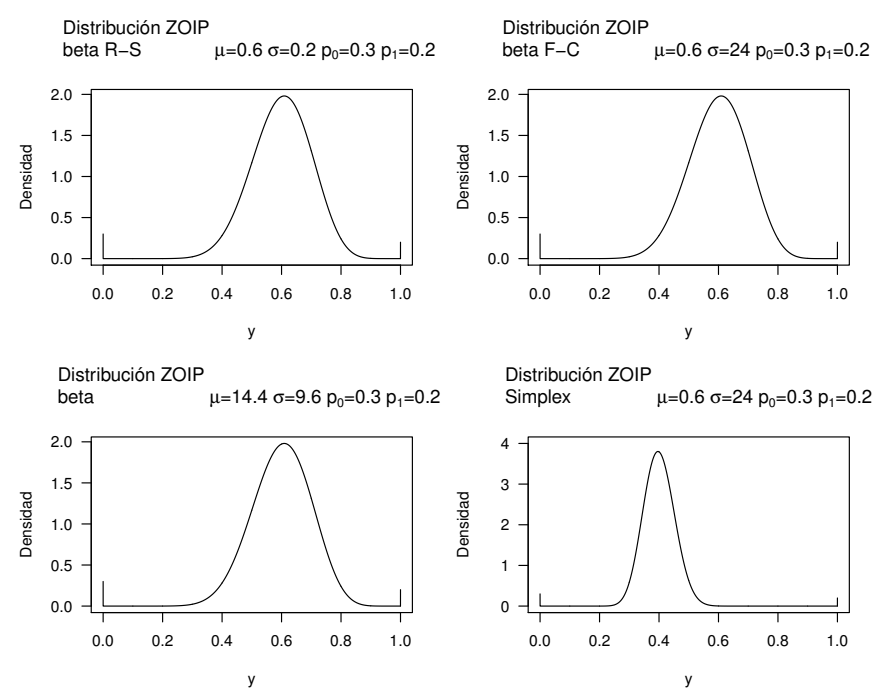

Figura 1: Densidades para la distribución ZOIP para algunos valores de los parámetros, donde $R$-S se refiere a Rigby $\&$ Stasinopoulos (2005) y F-C es Ferrari 85 Cribari-Neto (2004).

En la Figura 1 se muestran las densidades para algunas distribuciones ZOIPbeta en sus diferentes parametrizaciones y ZOIP-simplex, cabe aclarar que, en las figuras ubicadas en la esquina superior izquierda, esquina superior derecha y esquina inferior izquierda, los valores de los parámetros son diferentes, pero dan como resultado la misma distribución gracias a las ecuaciones descritas en (7), (8) para el caso Ferrari \& Cribari-Neto (2004) y (12), (13) para el caso Rigby \& Stasinopoulos (2005). Además se puede observar en la figura inferior derecha cómo la distribución ZOIP-simplex hereda el comportamiento bimodal de la distribución clásica simplex, con valores mínimo y máximo en cero y uno respectivamente.

\section{Inferencia estadística}

Para estimar los parámetros de la distribución ZOIP se usa el método de máxima verosimilitud. La función de verosimilitud para $\boldsymbol{\theta}=\left(\mu, \sigma, p_{0}, p_{1}\right)^{\top}$, basado en una muestra de $\boldsymbol{y}_{i}$ observaciones independientes, es de la forma:

$$
L(\boldsymbol{\theta})=\prod_{i=1}^{n} g\left(y_{i} ; \mu, \sigma, p_{0}, p_{1}\right)
$$

Para encontrar los estimadores de máxima verosimilitud (MLE) de la distribución ZOIP, se consideraran dos casos: 


\section{ZOIP-beta}

Considera la parametrización de la distribución beta y la ecuación definida en 20) se tiene que:

$$
\begin{gathered}
\boldsymbol{\theta}=\left(p, q, p_{0}, p_{1}\right)^{\top} \\
L(\boldsymbol{\theta})=\prod_{i=1}^{n} g\left(\boldsymbol{\theta} \mid y_{i}\right)=L_{1}\left(p_{0}\right) \cdot L_{2}\left(p_{1}\right) \cdot L_{3}(p, q)
\end{gathered}
$$

Note que la función de verosimilitud es factorizada en tres términos, dos de ellos del componente discreto y uno compuesto por $p$ y $q$ del componente continuo, por tanto, los parámetros son separables (Pace \& Salvan 1997), así la función verosimilitud puede ser tratada por separado.

$$
L_{1}\left(p_{0}\right)=\prod_{i=1}^{n} p_{0}^{S_{0}\left(y_{i}\right)}\left(1-p_{0}\right)^{1-S_{0}\left(y_{i}\right)}=p_{0}^{\sum_{i=1}^{n} S_{0}\left(y_{i}\right)}\left(1-p_{0}\right)^{n-\sum_{i=1}^{n} S_{0}\left(y_{i}\right)}
$$

Donde:

$$
S_{0}\left(y_{i}\right)=\left\{\begin{array}{ll}
1 & \text { si } y_{i}=j \\
0 & \text { si } y_{i} \neq j
\end{array} \quad ; \quad j=1,2\right.
$$

Ahora, al aplicar logaritmo natural a la función de verosimilitud se obtiene que:

$$
\hat{p}_{0}=\frac{1}{n} \sum_{i=1}^{n} S_{0}\left(y_{i}\right)
$$

Análogamente, se tiene que:

$$
\hat{p}_{1}=\frac{1}{n} \sum_{i=1}^{n} S_{1}\left(y_{i}\right)
$$

Ahora se halla el estimador de MV para los parámetros del componente continuo de la función.

$$
\begin{aligned}
\ell_{3}(p, q)= & \sum_{\left\{i: y_{i} \in(0,1)\right\}} \log \left(f\left(p, q \mid y_{i}\right)\right)=n \log (\Gamma(p+q))-n \log (\Gamma(p))-n \log (\Gamma(q)) \\
& +(p-1) \sum_{\left\{i: y_{i} \in(0,1)\right\}} \log \left(y_{i}\right)+(q-1) \sum_{i=1: y_{i} \in(0,1)}^{n} \log \left(1-y_{i}\right)
\end{aligned}
$$

entonces

$$
\frac{\partial \ell_{3}(p, q)}{\partial p}=\sum_{\left\{i: y_{i} \in(0,1)\right\}} \log \left(y_{i}\right)+\frac{n \cdot \partial \log (\Gamma(p+q))}{\partial p}-\frac{n \cdot \partial \log (\Gamma(p))}{\partial p}-\frac{n \cdot \partial \log (\Gamma(q))}{\partial p}=0
$$




$$
\begin{gathered}
\frac{\partial \ell_{3}(p, q)}{\partial q}=\sum_{\left\{i: y_{i} \in(0,1)\right\}} \log \left(1-y_{i}\right)+\frac{n \cdot \partial \log (\Gamma(p+q))}{\partial q}-\frac{n \cdot \partial \log (\Gamma(p))}{\partial q}-\frac{n \cdot \partial \log (\Gamma(q))}{\partial q}=0 \\
\frac{\partial \ell_{3}(p, q)}{\partial p}=\sum_{\left\{i: y_{i} \in(0,1)\right\}} \log \left(y_{i}\right)-n(-\psi(p+q)+\psi(p))=0 \\
\frac{\partial \ell_{3}(p, q)}{\partial q}=\sum_{\left\{i: y_{i} \in(0,1)\right\}} \log \left(1-y_{i}\right)-n(-\psi(p+q)+\psi(q))=0
\end{gathered}
$$

Donde $\psi(\cdot)=\Gamma^{\prime}(\cdot) / \Gamma(\cdot)$

Este sistema de ecuaciones no tiene una solución de forma cerrada, por lo que para encontrar los MLE de $p$ y $q$ es necesario utilizar algoritmos iterativos, por ejemplo el método de Newton Raphson, mínimos cuadrados ponderados. En el paquete ZOIP se utiliza optimizadores a la función de verosimilitud mediante la función nlminb o optim de R, basadas en las funciones de Fortran drmnfb, drmngb, drmnhb y el metodo de Nelder y Mead, respectivamente, sin embargo, se puede garantizar que los puntos críticos encontrados serán máximos de la función de verosimilitud, ya que si hallamos la segunda derivada de la función se tiene que:

$$
\begin{aligned}
& \frac{\partial^{2} \ell_{3}(p, q)}{\partial p^{2}}=-n\left(\psi^{\prime}(p)-\psi^{\prime}(p+q)\right)<0 \\
& \frac{\partial^{2} \ell_{3}(p, q)}{\partial q^{2}}=-n\left(\psi^{\prime}(q)-\psi^{\prime}(p+q)\right)<0
\end{aligned}
$$

Lo anterior debido que la varianza de la transformación logarítmica de la variable es:

$$
\begin{gathered}
\operatorname{var}(\log (y))=E\left[\log ^{2}(y)\right]-(E[\log (y)])^{2}=\psi^{\prime}(p)-\psi^{\prime}(p+q)>0 \\
\operatorname{var}(\log (1-y))=E\left[\log ^{2}(1-y)\right]-(E[\log (1-y)])^{2}=\psi^{\prime}(q)-\psi^{\prime}(p+q)>0
\end{gathered}
$$

ver más en Owen (2008).

Para encontrar las estimaciones de los parámetros de beta en parametrizaciones de Ferrari \& Cribari-Neto (2004) y Rigby \& Stasinopoulos (2005), basta con encontrar los estimadores MLE anteriores de la parametrización clásica y utilizar las ecuaciones definidas en (7), (8) para el caso de Ferrari \& Cribari-Neto (2004) y (12, 13 para el caso de Rigby \& Stasinopoulos (2005).

\section{ZOIP-simplex}

Comunicaciones en Estadística, junio 2018, Vol. 11, No. 1 
Para este caso la única diferencia con respecto al caso anterior es la estimación en el componente continuo, por lo tanto.

$$
L_{3}(\mu, \sigma)=\prod_{\left\{i: y_{i} \in(0,1)\right\}}\left[2 \pi \sigma^{2}\left[y_{i}\left(1-y_{i}\right)\right]^{3}\right]^{-1 / 2} \exp \left(-\frac{1}{2 \sigma^{2}} d\left(y_{i} ; \mu\right)\right),
$$

donde $d\left(y_{i} ; \mu\right)=\frac{y_{i}\left(1-y_{i}\right) \mu^{2}(1-\mu)^{2}}{\left(y_{i}-\mu\right)^{2}}$.

$\ell_{3}(\mu, \sigma)=-\frac{n}{2} \log (2 \pi)-\frac{n}{2} \log \left(\sigma^{2}\right)-\frac{3}{2} \sum_{\left\{i: y_{i} \in(0,1)\right\}} \log \left(y_{i}\left(1-y_{i}\right)\right)-\sum_{\left\{i: y_{i} \in(0,1)\right\}} \frac{1}{2 \sigma^{2}} d\left(y_{i} ; \mu\right)$

$\frac{\partial \ell_{3}(\mu, \sigma)}{\partial \sigma}=-\frac{n}{\sigma}+\frac{1}{\sigma^{3}} \sum_{\left\{i: y_{i} \in(0,1)\right\}} d\left(y_{i} ; \mu\right)=\sigma\left(-n \sigma^{2}+\sum_{\left\{i: y_{i} \in(0,1)\right\}} d\left(y_{i} ; \mu\right)\right)=0$

El valor de $\sigma=0$ no es posible, por lo tanto:

$$
\begin{gathered}
-n \sigma^{2}+\sum_{i=1: y_{i} \in(0,1)}^{n} d\left(y_{i} ; \mu\right)=0 \\
\therefore \hat{\sigma}^{2}=\frac{1}{n} \sum_{i=1: y_{i} \in(0,1)}^{n} d\left(y_{i} ; \hat{\mu}\right)
\end{gathered}
$$

El estimador MLE de $\sigma^{2}$ depende del valor estimado en $\mu$, entonces:

$$
\frac{\partial \ell_{3}(\mu, \sigma)}{\partial \sigma}=-\frac{1}{2 \sigma^{2}} \sum_{i=1: y_{i} \in(0,1)}^{n} \frac{\partial d\left(y_{i} ; \mu\right)}{\partial \mu}=0
$$

Donde

$$
\begin{aligned}
\frac{\partial d\left(y_{i} ; \mu\right)}{\partial \mu}=\sum_{i=1: y_{i} \in(0,1)}^{n} & \frac{y_{i}\left(1-y_{i}\right) \mu^{2}(1-\mu)^{2}}{2\left(y_{i}-\mu\right)^{3}} \\
+ & \frac{2 y_{i}\left(1-y_{i}\right) \mu(1-\mu)^{2}-2 y_{i}\left(1-y_{i}\right) \mu^{2}(1-\mu)}{\left(y_{i}-\mu\right)^{2}}=0
\end{aligned}
$$

No tiene una solución cerrada analíticamente, entonces se deben utilizar algoritmos iterativos tal como Newton Raphson o mínimos cuadrados ponderados, en el paquete ZOIP se utiliza optimizadores para la función de verosimilitud mediante la función nlminb o optim de R, para encontrar puntos críticos donde $\partial d\left(y_{i} ; \mu\right) / \partial \mu=0$. 


\section{Paquete ZOIP}

En esta sección se presenta el paquete ZOIP de R alojado en el CRAN y en GitHub y creado por los autores para analizar datos proporcionales inflados con ceros y/o unos y ajustar una distribución ZOIP.

\subsection{Instalación}

Para acceder a la última versión del paquete ZOIP se puede ingresar al repositorio CRAN, donde se encuentra disponible. Otra opcion es instalar la version en desarrollo desde GitHub, el cual es un alojamiento de repositorios Git, para obtener dicha versión es necesario ejecutar el siguiente código que instala el paquete devtools, que es necesario para descargar el paquete ZOIP desde GitHub.

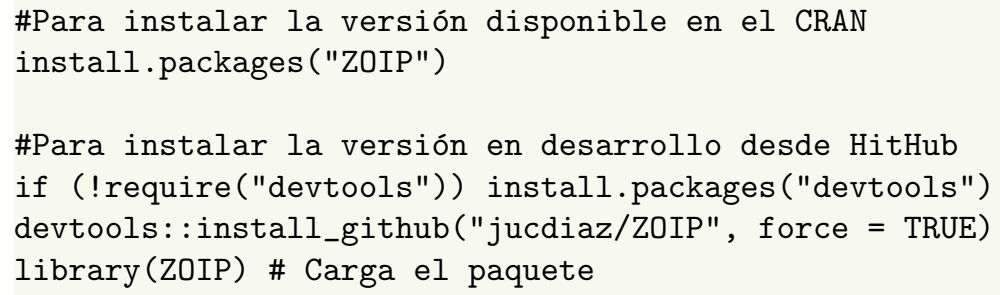

\subsection{Funciones sobre distribución ZOIP}

En el paquete ZOIP existen cuatro funciones llamadas dZOIP, pZOIP, qZOIP y rZOIP que corresponden a las funciones de densidad de probabilidad, la función de distribución acumulada, la función cuantil y la función generadora de números aleatorios de la distribución ZOIP, respectivamente; estas cuatro funciones tendrán como argumentos los parámetros de la distribución y un argumento llamado family, el cual corresponderá al tipo de distribución para datos proporcionales a utilizar. En el siguiente código se observa cómo se halla la densidad de probabilidad en el punto 0.5 de una distribución ZOIP-beta con parametrización Rigby \& Stasinopoulos (2005) descrita como $\mathrm{ZOIP}\left(\mu=0.2, \sigma=0.5, p_{0}=0.2, p_{1}=0.2\right)$ :

$\operatorname{dZOIP}(\mathrm{x}=0.5, \mathrm{mu}=0.2, \operatorname{sigma}=0.5, \mathrm{p} 0=0.2, \mathrm{p} 1=0.2$, family='R-S' $)$ \#\#[1] 0.3243543

Además se halla la probabilidad acumulada hasta el punto 0.5 de una distribución OIP-beta con parametrización Ferrari \& Cribari-Neto (2004) dada por $\mathrm{ZOIP}\left(\mu=0.2, \sigma=3, p_{0}=0, p_{1}=0.2\right)$ : 
$\operatorname{pZOIP}(q=0.5, \mathrm{mu}=0.2, \operatorname{sigma}=3, \mathrm{p} 0=0, \mathrm{p} 1=0.2$, family='F-C' $)$

\#\#[1] 0.7181223

Se calcula el percentil $70 \%$ de una distribución ZIP-beta dada por $\operatorname{ZOIP}(\mu=$ $\left.0.6, \sigma=2.4, p_{0}=0.2, p_{1}=0\right)$ :

qZOIP $\left(\mathrm{p}=0.7, \mathrm{mu}=0.6\right.$, sigma $=2.4, \mathrm{p} 0=0.2, \mathrm{p} 1=0$, family='Original $\left.^{\prime}\right)$

\#\#[1] 0.2061418

Por último, se generan ocho valores aleatorios de una distribución ZOIP-simplex descrita como $\operatorname{ZOIP}\left(\mu=0.6, \sigma=3, p_{0}=0.2, p_{1}=0.2\right)$. La función set.seed tiene como fin garantizar la repetición de los valores aleatorios generados en el ejemplo.

set.seed (12345)

$\operatorname{rZOIP}(\mathrm{n}=8, \mathrm{mu}=0.2, \operatorname{sigma}=3, \mathrm{p} 0=0.2, \mathrm{p} 1=0.2$, family='Simplex' $)$

$\begin{array}{lllllll}\text { \#\#[1] } & 0.3185479 & 1.0000000 & 0.3765073 & 1.0000000 & 0.1626598\end{array}$

$\begin{array}{lllll}\text { \#\#[6] } & 0.0000000 & 0.1138673 & 0.1840670\end{array}$

\subsection{Estimación de parámetros}

La función RM.ZOIP estima los parámetros de una distribución ZOIP vía máxima verosimilitud utilizando el optimizador deseado (nlminb,optim). La estructura de la función RM.ZOIP es la siguiente:

RM.ZOIP (formula.mu, data, family = "R-S", optimizer="nlminb")

Los argumentos de la función RM.ZOIP son:

- formula.mu: formula que define los datos para ajustar una distibución ZOIP, para ello debe tomar el valor de y $\sim 1$, donde y es el nombre de la variable a ajustar.

- data: es el conjunto de datos en formato data.frame donde debe contener los datos de la variable a ajustar y el nombre debe ser el que está en las fórmula para el parámetro $\mu$.

- family: elección de la distribución ZOIP deseada para ajustar, si toma el valor de ' $R-S$ ') se utilizará la distribución ZOIP-beta con parametrización (Rigby \& Stasinopoulos 2005), si toma el valor de ' F-C' ' se utilizará la distribución ZOIP-beta parametrización (Ferrari \& Cribari-Neto 2004), en 
el valor de "'Original' ' se utilizará la distribución ZOIP-beta con parametrización clásica, "'Simplex" ' utilizará la distribución ZOIP-simplex.

- optimizer: elección del optimizador, utilizado para encontrar la convergencia de la máxima verosimilitud. se puede elegir el valor de " $n l m i n b "$ " o '"optim", por defecto "nlminb"'.

En el siguiente ejemplo se mostrará el ajuste de una distribución ZOIP, para ello, se mostrará la salida de la función RM.ZOIP de 1000 observaciones simuladas para la distribución ZOIP-beta parametrización (Rigby \& Stasinopoulos 2005).

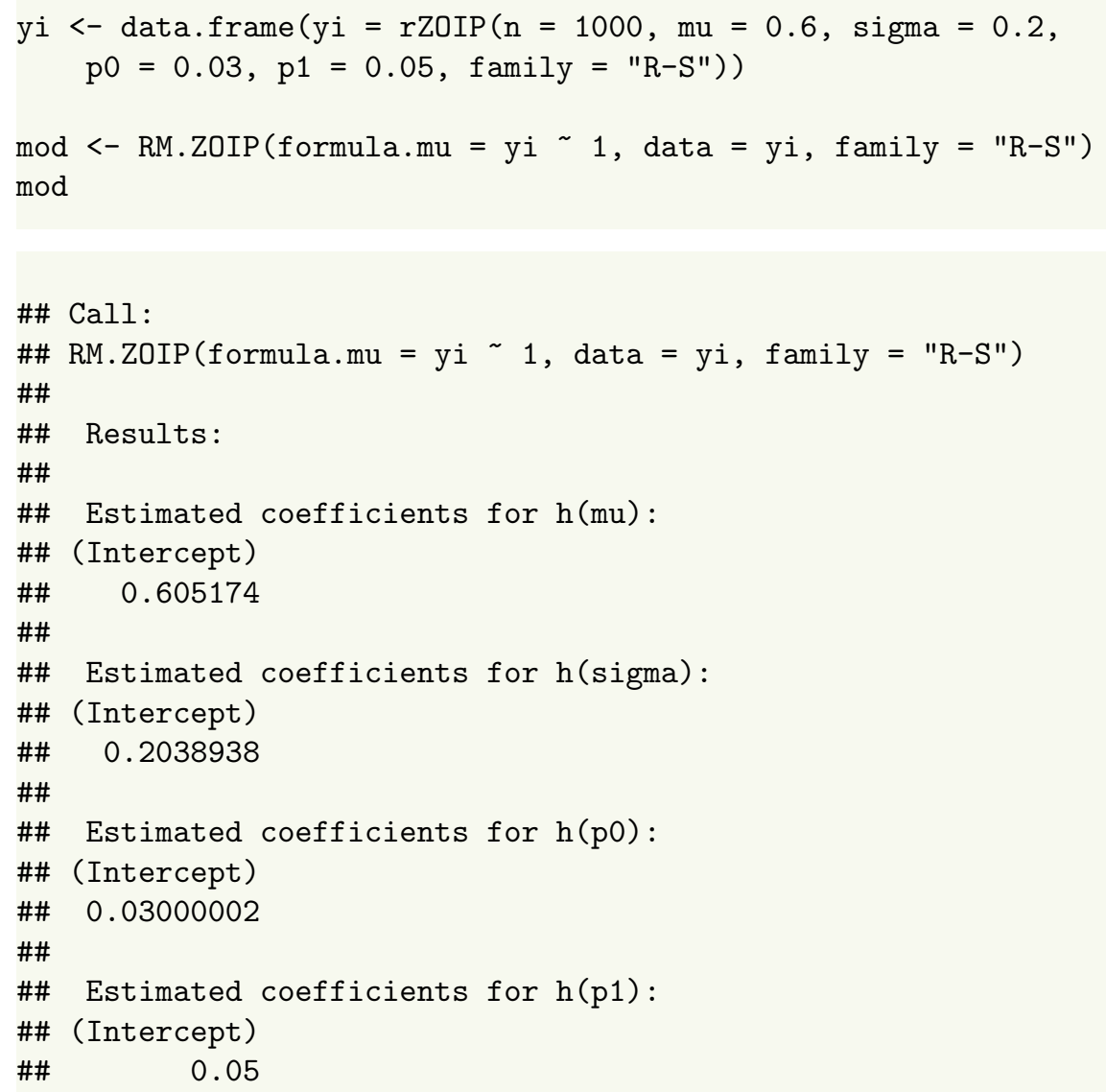




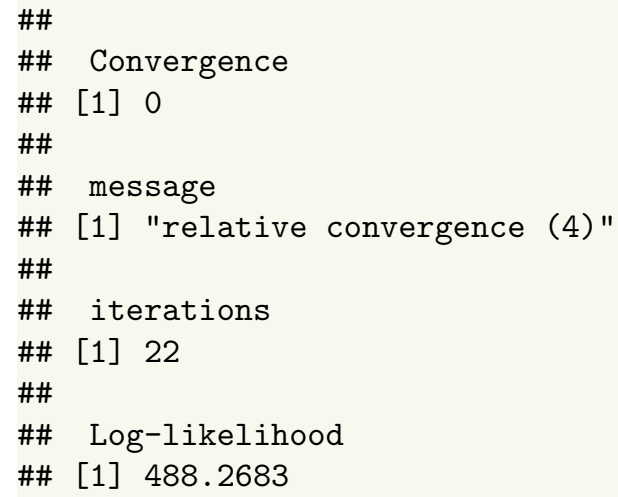

En el anterior resultado se obtienen varios aspectos importantes de la salida del ajuste de la distribución y leyendo de arriba hacia abajo, primero que todo nos muestra la distribución ajustada, luego el valor ajustado para cada parámetro de la distribución ZOIP, luego un indicador de convergencia del ajuste, donde 0 indica la convergencia, después un mensaje sobre la convergencia (resultados heredados de la función nlimnb), después se encuentra el número de iteraciones que fueron necesarias para que convergiera el ajuste de la distribución, por último, encuentra valor de la log-verosimilitud que nos permitirá hacer comparaciones entre ajustes de distribuciones.

Al aplicar a la distribución ajustada (mod) la función summary se obtiene el siguiente resultado:

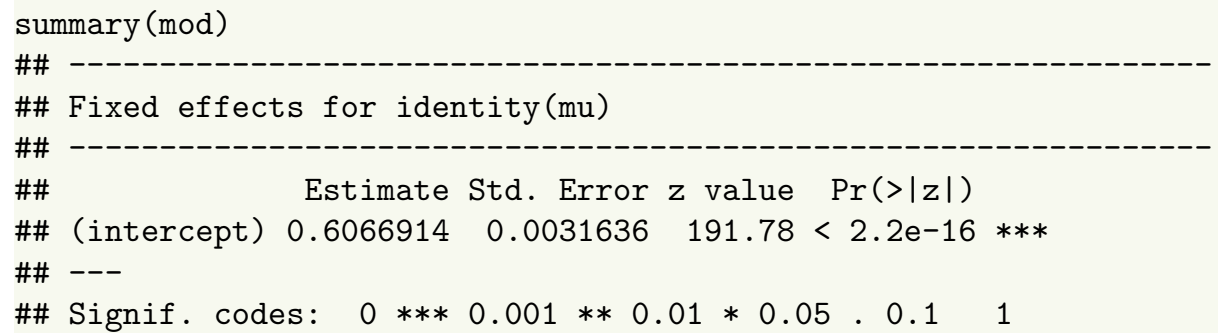




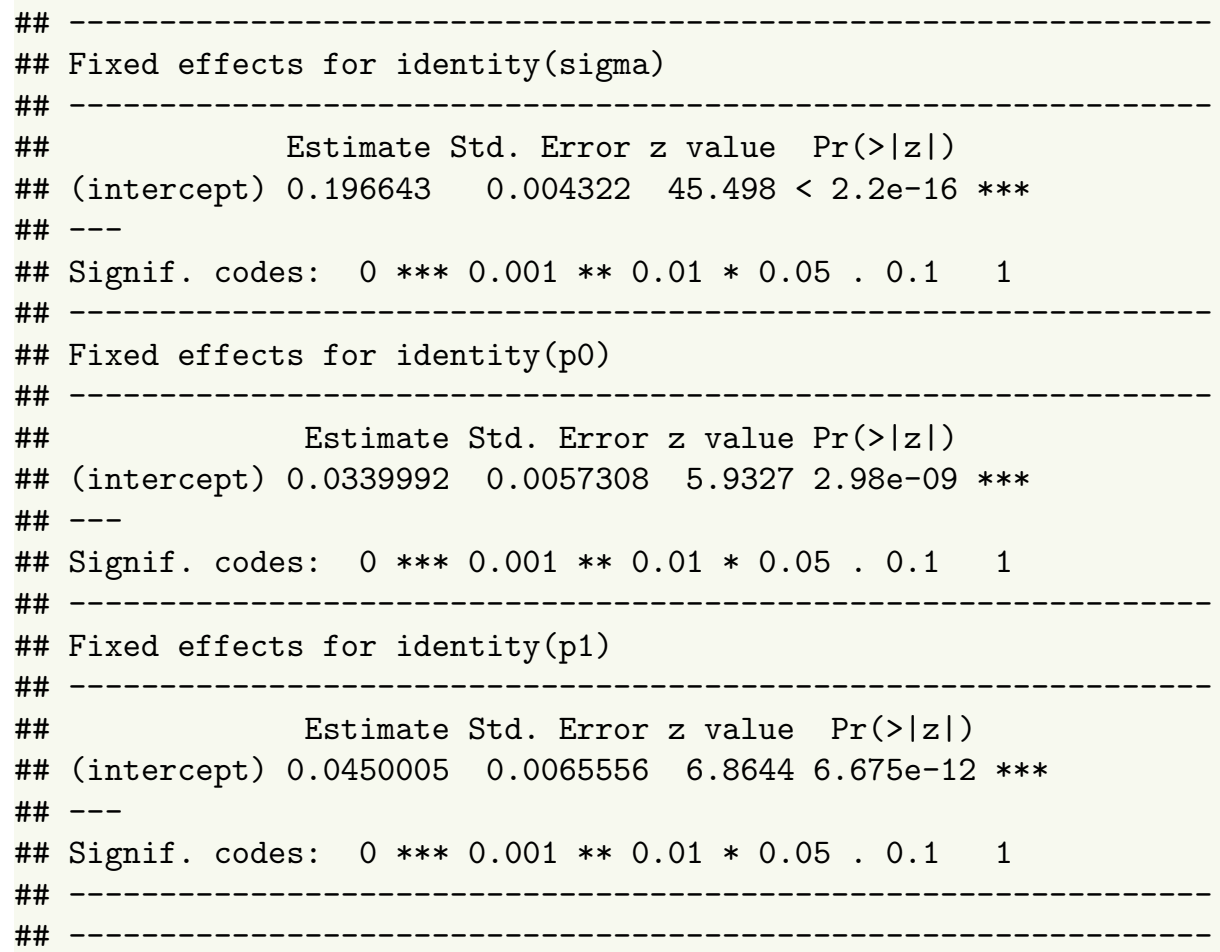

Con la función summary aplicada a la distribución ZOIP ajustada, se obtiene más detalles de los parámetros estimados, primero se obtiene el valor estimado (Estimate), su error estándar (Std.Error), el valor $\mathrm{Z}$ del estimador ( $\mathrm{z}$ value) y el valor p que indicará la significancia del parámetro estimado $(\operatorname{Pr}(>|z|))$.

En el resultado anterior se obtienen los valores de $\hat{\mu}=0.6066914, \hat{\sigma}=0.196643$, $\hat{p}_{0}=0.0339992$ y $\hat{p}_{1}=0.0450005$, que son similares a los parámetros con los que se simuló $y_{i}$. Además, cabe resaltar que en la función RM. ZOIP para ajustar distribuciones de probabilidad no es necesario colocar funciones de enlace ni espacio de busqueda de los parámetros, ya que estos son introducidas automáticamente de acuerdo con el valor tomado en family.

Así como la función summary puede ser aplicada a un objeto de la clase ZOIP, en este trabajo se implementaron otros tipo de funciones asociadas a métodos S3 de $\mathrm{R}$ a objetos de clase ZOIP, tales como la función print y coef, que permiten mostrar los resultados del modelo ajustado en general y los parámetros regresores estimados, respetivamente. 


\section{Estudio de simulación}

En esta sección se realiza un estudio de simulación para observar la convergencia de la estimación de los parámetros de la distribución ZOIP, para esto se analizan diferentes aspectos de la capacidad de estimación que tiene el método de máxima verosimilitud sobre los parámetros de la distribución ZOIP. Se generan muestras de una distribución ZOIP bajo las diferentes distribuciones y parámetrizaciones con tamaños de muestra $n$ de: $5,10,15, \ldots, 500$, y se realizaron 1000 réplicas para cada tamaño de muestra, posteriormente se calcula la mediana de cada una de las estimaciones de los parámetros, y así poder analizar la capacidad de convergencia de las metodologías implementadas en la distribución ZOIP y en el paquete ZOIP.

En el primer escenario del estudio de simulación se generan los datos de una distribución ZOIP-beta $\left(\mu=0.6, \sigma=0.2, p_{0}=0.03, p_{1}=0.05\right)$ para el caso de la parametrización de Rigby \& Stasinopoulos (2005), ZOIP-beta $(\mu=0.6, \sigma=$ $\left.24, p_{0}=0.03, p_{1}=0.05\right)$ para el caso de la parametrización de Ferrari \& CribariNeto (2004), ZOIP-beta $\left(\mu=14.4, \sigma=9.6, p_{0}=0.03, p_{1}=0.05\right)$, cabe aclarar que las tres parametrizaciones anteriores generan exactamente la misma distribución, esto gracias a las ecuaciones definidas en (7), (8), (12) y (13); de igual manera se generó la misma cantidad de datos simulados para la distribución ZOIP$\operatorname{simplex}\left(\mu=0.4, \sigma=0.2, p_{0}=0.03, p_{1}=0.05\right)$.

En la Figura 2 se presentan las medianas de la estimación de los parámetros para cada tamaño de muestra, de esta figura se observa que independientemente de la distribución y parametrización escogida en la distribución ZOIP, todas las estimaciones convergen en el valor verdadero del parámetro a medida que aumenta el tamaño de muestra $n$. En la Figura 2 se nota que las estimaciones de $\sigma$ cuando son parámetros con significado de dispersión como es en la distribución Beta con parametrización Rigby \& Stasinopoulos (2005) y en la distribución simplex, tienden a dar valores subestimados, por otra parte, en las distribuciones donde $\sigma$ tiene significado de forma y precisión tienden a dar valores sobrestimados. Se observa que las estimaciones de los parámetros de inflación, a pesar de que son pequeñas, dan resultados muy satisfactorios y casi sin variación en su forma de estimación de distribución a distribución.

Como medida global del proceso de estimación se eligió el MAPE (error porcentual absoluto medio, $\left.\left(\left(\sum_{i=1}^{n}\left|y_{i}-\hat{y}_{i} / y_{i}\right|\right) / n\right)\right)$ debido a los cambios de escala entre los diferentes parámetros de las diferentes distribuciones y parametrizaciones. Esta media se realizó como un promedio de los MAPES generados por cada uno de los parámetros de la distribución ZOIP en cada tamaño de muestra. En la Figura 4a se presenta el MAPE para las diferentes distribuciones y parametrizaciones estimadas, se observa como a medida de que el tamaño de muestra aumenta, el MAPE va decreciendo rápidamente, aunque después de un tamaño de muestra de 200, el MAPE decrece de una manera más lenta, además los errores de estimación son muy parecidos entre los cuatro casos de simulación, la estimación sobre los parámetros de la distribución ZOIP-simplex tiene un error un poco más grande, 
54Juan Camilo Díaz Zapata, Freddy Hernández Barajas \& Olga Cecilia Usuga Manco
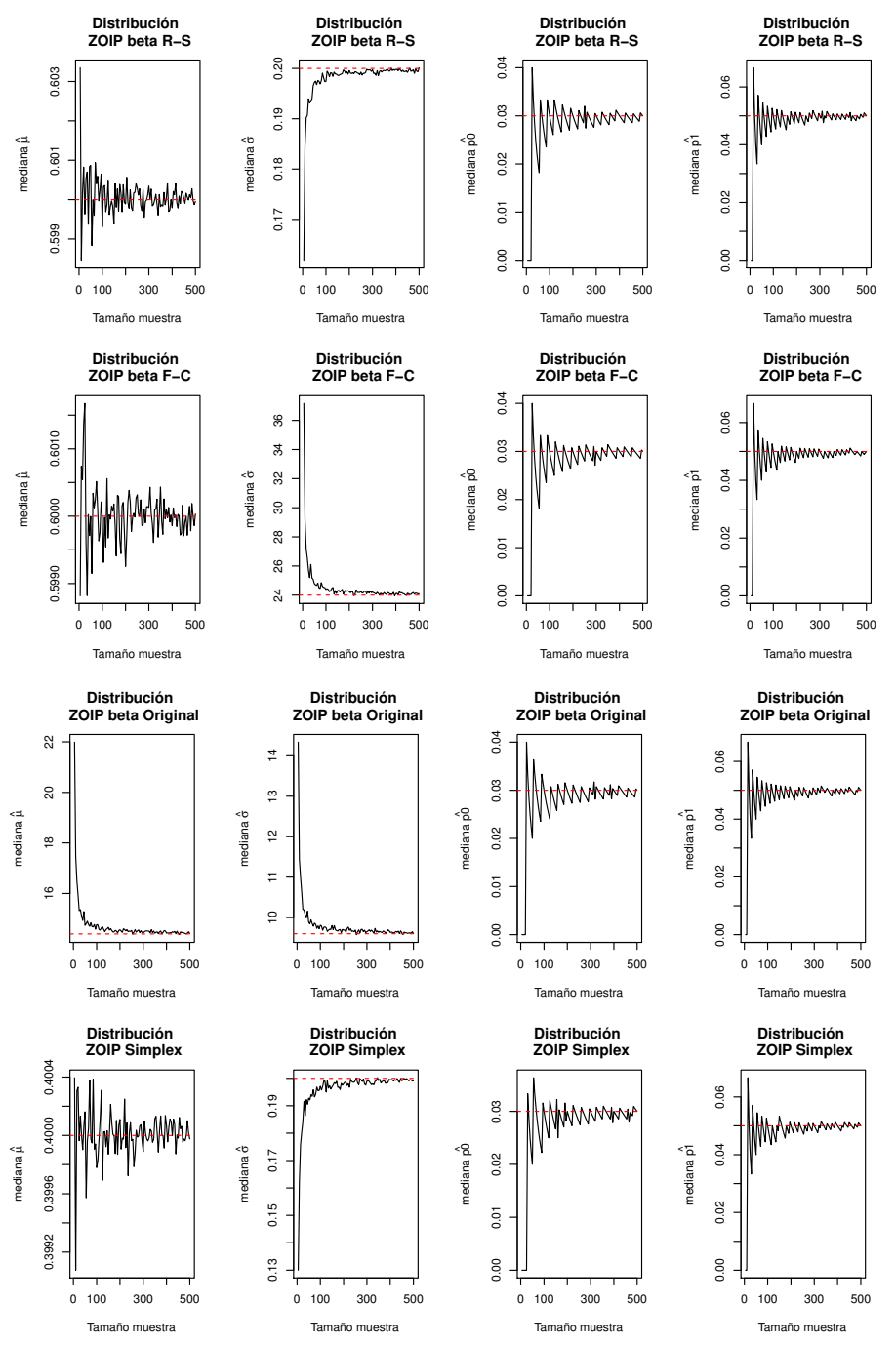

Figura 2: Mediana de los parámetros estimados en el escenario 1 para distintas parametrizaciones y valores de $n$, las líneas punteadas representan el verdadero valor del parámetro.

pero no es significativo sobre los demás casos.

En el segundo escenario de simulación se generó el mismo ejercicio de simulación anterior sobre las mismas distribuciones y parametrizaciones, solo que los valores de $p_{0}$ y $p_{1}$ cambian por 0.3 y 0.2 , respectivamente. Dando así que el $50 \%$ de los datos se vean contaminados por ceros y unos, esto para ver si de alguna forma 
afecta el aumento de la presencia de ceros y unos sobre las estimaciones de los parámetros de la parte continua de la distribución ZOIP.
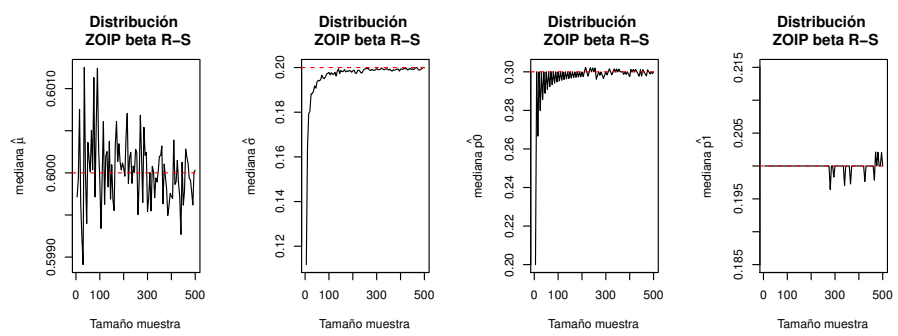

Distribución
ZoIP beta F-C
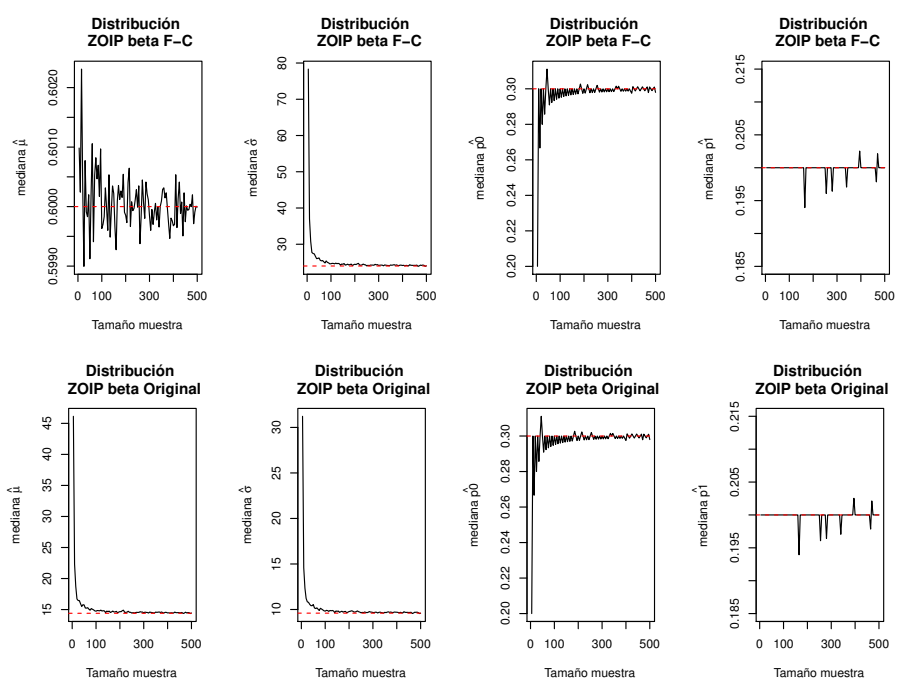

Distribución
ZOIP Simplex
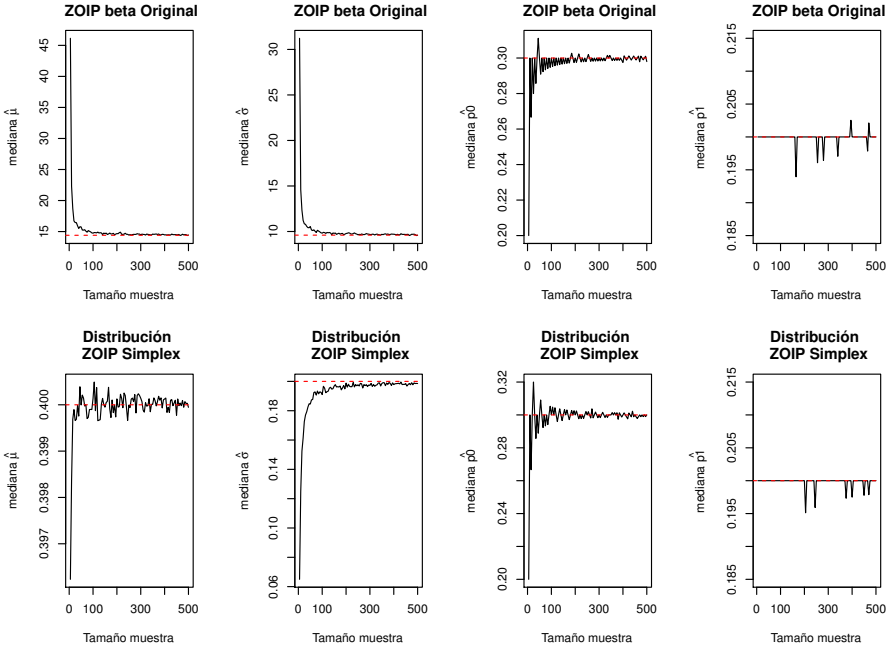

Figura 3: Simulación de distribución ZOIP para distintas parámetrizaciones con parámetros de inflación grandes, distribuciones y valores de $n$.

En la Figura 3 se presentan las estimaciones de los parámetros de la simulación con inflaciones al $50 \%$ para diferentes tamaños de muestras, en general, no se observan cambios muy significativos sobre la Figura 2 en los parámetros de $\mu$ y $\sigma$, sin embargo, en la estimación de $p_{0}$ se tienden a dar valores subestimados con relación al estudio de simulación anterior y con el parámetro $p_{1}$, aunque las estimaciones 
56Juan Camilo Díaz Zapata, Freddy Hernández Barajas \& Olga Cecilia Usuga Manco
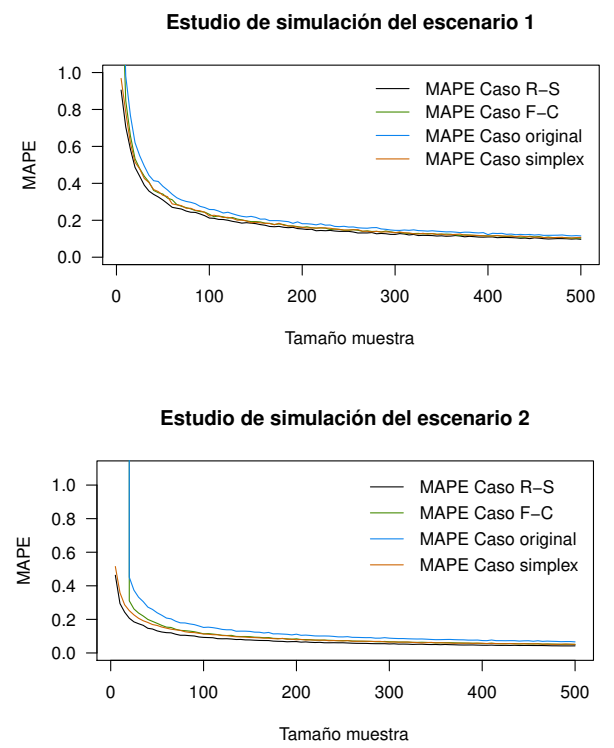

Figura 4: Mape (Error porcentual absoluto medio) para los dos escenarios de simulación y para distintas parametrizaciones y valores de $n$.

son muy acertadas sobre el valor real desde tamaños de muestra pequeños, en algunas ocasiones se producen pequeñas perturbaciones no muy alejados del valor real.

Tabla 2: MAPE de las estimaciones para cada parámetro en diferentes parametrizaciones en los dos estudios de simulación.

\begin{tabular}{|c|l|cc|}
\hline Parámetro & Caso & MAPE escenario 1\% & MAPE escenario 2 \% \\
\hline \multirow{5}{*}{$\mu$} & ZOIP-Beta R-S & 0.61 & 0.86 \\
& ZOIP-Beta F-C & 0.50 & 0.85 \\
& ZOIP-Beta & 0.53 & 0.70 \\
& simplex & 0.47 & 0.63 \\
\hline \multirow{5}{*}{$\sigma$} & ZOIP-Beta R-S & 2.53 & 3.40 \\
& ZOIP-Beta F-C & 5.10 & 6.90 \\
& ZOIP-Beta & 5.30 & 6.98 \\
& simplex & 5.30 & 7.37 \\
\hline \multirow{5}{*}{$p_{0}$} & ZOIP-Beta R-S & 20.5 & 5.36 \\
& ZOIP-Beta F-C & 19.7 & 5.42 \\
& ZOIP-Beta & 19.8 & 5.43 \\
& simplex & 20.8 & 5.51 \\
\hline \multirow{5}{*}{$p_{1}$} & ZOIP-Beta R-S & 15.2 & 7.28 \\
& ZOIP-Beta F-C & 16.0 & 7.00 \\
& ZOIP-Beta & 15.7 & 7.00 \\
& simplex & 16.2 & 7.12 \\
\hline & Promedio & 10.57 & 5.26 \\
\hline
\end{tabular}

En la Figura 4b se presenta el MAPE para el estudio de simulación del segundo

Comunicaciones en Estadística, junio 2018, Vol. 11, No. 1 
escenario, se puede ver cómo se obtienen MAPES muy parecidos a los del estudio de simulación del primer escenario, pero cabe resaltar como se comete menos error sobre la estimación de los parámetros de la distribución beta con parametrización Rigby \& Stasinopoulos (2005). En la Tabla 2 se presenta el MAPE para el parámetro de cada parametrización de ambos estudios de simulación, es claro ver cómo en general el estudio de simulación del segundo escenario produce un MAPE menor que el del primer escenario, esto es causado porque en el primer escenario de simulación los errores de pronóstico son más grandes en los parámetros de inflación que en el segundo escenario. Por todo lo visto anteriormente se puede concluir que el crecimiento de los parámetros de inflación no afecta de manera significativa la estimación de los parámetros de la parte continua de la distribución ZOIP, pero sí en una mejor estimación de los parámetros de inflación.

\section{Aplicación a datos reales}

En esta sección se presenta el ajuste de una distribución ZOIP a datos reales sobre la utilización de una tarjeta de crédito en un banco.

Para una entidad financiera grande como un banco es de vital importancia conocer el comportamiento del porcentaje de utilización de sus tarjetas de crédito (tdc), se define a $y$ como el porcentaje de uso de una tdc (saldo utilizado sobre cupo total de la tdc), en la Figura 5 se presenta el histograma del porcentaje de utilización de las tdc y es claro notar que $y$ se encuentra entre cero y uno, pero adicionalmente es muy común ver que las tdc no sean utilizadas $(y=0)$ y también que las tdc sean utilizadas en la totalidad de su cupo asignado $(y=1)$, por lo que se trata a $y$ como una variable aleatoria perteneciente a datos proporcionales inflados con ceros y unos. Se tiene un total de 9206 tdc, que representan el porcentaje de utilización de las tdc para un trimestre de 2014 en el banco. Se quiere estudiar el ajuste de una distribución ZOIP, para ello se utiliza el paquete en R llamado ZOIP mediante su función RM.ZOIP.

En la Tabla 3 se muestran resultados de los cuatro parámetros estimados vía máxima verosimilitud para la distribución ZOIP, en ellas se ve cómo cambian los valores de los parámetros según la parametrización escogida, los valores de logverosimilitud indican que el mejor modelo ajustado es un ZOIP-beta, ya que es bastante menor el valor de log-verosimilitud de una distribución ZOIP-simplex; además, en las estimaciones de los parámetros de la distribución ZOIP-simplex no se tuvo una convergencia, por lo tanto, los valores son muy distintos para el parámetro de dispersión a los vistos en la distribución ZOIP-beta, inclusive muy elevados. Por otro lado, el valor de $\mu$ es mayor que las de la parametrización en Rigby \& Stasinopoulos (2005) y Ferrari \& Cribari-Neto (2004), un 17 \% más.

En la Figura 6 se presenta gráficamente el ajuste de la distribución ZOIP-beta 
58Juan Camilo Díaz Zapata, Freddy Hernández Barajas \& Olga Cecilia Usuga Manco

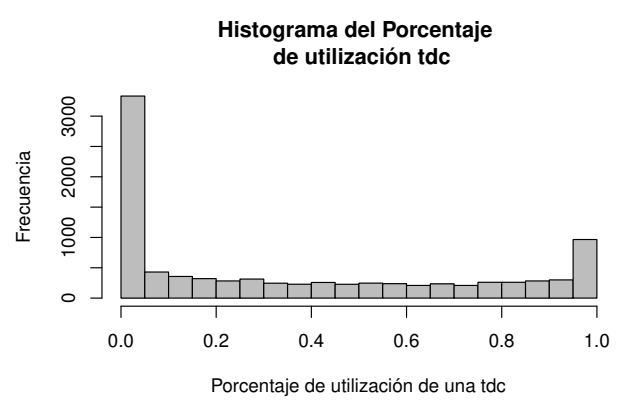

Figura 5: Histograma del porcentaje de utilización de las tdc en un banco.
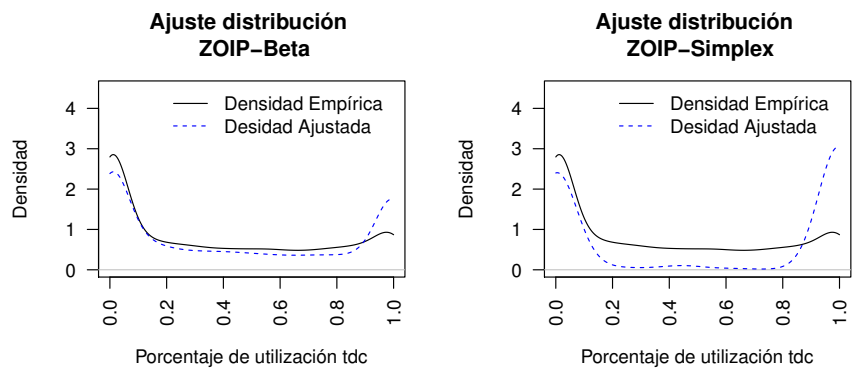

Figura 6: Ajuste de diferentes distribuciones y parametrizaciones ZOIP al porcentaje de utilización de una tdc. 
Tabla 3: Ajuste de diferentes distribuciones ZOIP en el porcentaje de utilización de una tdc.

\begin{tabular}{|c|c|ccc|c|}
\hline Familia & Parámetro & Estimación & Error estándar & Valor P & Log-Verosimilitud \\
\hline \multirow{4}{*}{ R-S } & $\mu$ & 0.4040 & 0.0037 & $<2.2 e^{-16}$ & \\
& $\sigma$ & 0.6601 & 0.0027 & $<2.2 e^{-16}$ & 5854.067 \\
& $p_{0}$ & 0.2219 & 0.0043 & $<2.2 e^{-16}$ & \\
& $p_{1}$ & 0.0695 & 0.0027 & $<2.2 e^{-16}$ & \\
F-C & $\mu$ & 0.4040 & 0.0037 & $<2.2 e^{-16}$ & \\
& $\sigma$ & 0.4040 & 0.0037 & $<2.2 e^{-16}$ & 5854.067 \\
& $p_{0}$ & 0.2219 & 0.0043 & $<2.2 e^{-16}$ & \\
\hline \multirow{5}{*}{ original } & $p_{1}$ & 0.0695 & 0.0027 & $<2.2 e^{-16}$ & \\
& $\mu$ & 0.5233 & 0.0080 & $<2.2 e^{-16}$ & \\
& $\sigma$ & 0.7719 & 0.0130 & $<2.2 e^{-16}$ & 5854.067 \\
& $p_{0}$ & 0.2219 & 0.0043 & $<2.2 e^{-16}$ & \\
simplex & $p_{1}$ & 0.0695 & 0.0027 & $<2.2 e^{-16}$ & \\
& $\mu$ & 0.5741 & 0.0010 & $<2.2 e^{-16}$ & \\
& $\sigma$ & 4885.4370 & 18.2430 & $<2.2 e^{-16}$ & 54425.63 \\
& $p_{0}$ & 0.1497 & 0.0032 & $<2.2 e^{-16}$ & \\
\hline
\end{tabular}

a la izquierda y de la distribución ZOIP-simplex a la derecha sobre la densidad empírica del porcentaje de utilización de las tdc, la línea punteada representa la distribución ZOIP ajustada, cabe resaltar que el ajuste de la distribución ZOIPbeta es igual en las tres parametrizaciones planteadas, por lo que se presenta una sola gráfica para las tres parametrizaciones, se ve cómo la distribución ZOIP-beta sigue el comportamiento original del porcentaje de utilización de las tdc. Es bueno resaltar también que la distribución ZOIP-simplex no tiene un buen ajuste para los valores entre cero y uno. Por todo anterior es recomendable decir que el porcentaje de utilización de las tdc de este banco se comportan como una distribución ZOIPbeta con los parámetros descritos en la Tabla 3 según la parametrización deseada y no como una distribución ZOIP-simplex.

\section{Conclusiones}

La distribución ZOIP y el paquete ZOIP de R permiten ajustar distribuciones para datos provenientes de porcentajes, tasas o proporciones que se encuentren inflados con ceros y unos, dicha distribución está compuesta por cuatro parámetros, que son estimados vía máxima verosimilitud y en el cual, de acuerdo con los estudios de simulación realizados, estos convergen a los valores reales con un tamaño de muestra relativamente pequeño; además, se observa cómo la estimación de los parámetros de la parte continua no se ven afectados por el aumento de la presencia de ceros y unos en los datos, pero sí la estimación de los parámetros de la parte discreta. Por otra parte, se observa cómo el ajuste de la distribución ZOIP-beta explica el comportamiento de la distribución del porcentaje de utilización de una tarjeta de crédito en un banco. 
La distribución ZOIP y el paquete ZOIP de R permiten de una manera muy versátil utilizar y ajustar diferentes parametrizaciones y distribuciones para datos proporcionales. Permite también utilizar y ajustar distribuciones para datos proporcionales que se encuentran inflados solo con ceros o solo con unos, de una manera práctica.

Recibido: 31 de mayo de 2017

Aceptado: 28 de mayo de 2018

\section{Referencias}

Cepeda-Cuervo, E., Jaimes, D., Marin, M. \& Rojas, J. (2016), 'Bayesian beta regression with bayesianbetareg R-package', Computational Statistics 31(1), 165-187.

Ferrari, S. \& Cribari-Neto, F. (2004), 'Beta regression for modelling rates and proportions', Journal of applied statistics 31(7), 799-815.

Galvis, D. \& Lachos, V. (2014), Bayesian analysis of regression models for proportional data in the presence of zeros and ones, $\mathrm{PhD}$ thesis, Estatal University of Campinas.

Hahn, E. (2008), 'Mixture densities for project management activity times: A robust approach to pert', European Journal of operational research 188(2), 450459 .

Hossain, A. (2015), 'Modelling a proportion response variable using generalized additive models for location scale and shape', International Workshop on Statistical Modelling 2, 137-140.

Jørgensen, B. (1997), 'The theory of dispersion models', Computational statistics and Data analysis $\mathbf{7 6}$.

Jørgensen, B. \& Barndorff-Nielsen (1991), 'Some parametric models on the simplex', Journal of multivariate analysis 39(1), 106-116.

Kosmidis, I., Zou, A. \& Jagan, K. (2015), 'Beyond beta regression: modelling percentages and fractions in the presence of boundary observations', International Workshop on Statistical Modelling 1, 267-272.

Ospina, R. \& Ferrari, S. (2010), 'Inflated beta distributions online: doi:10.1007/s00362-008-0125-4', Statitical papers .

Ospina, R. \& Ferrari, S. (2012), 'A general class of zero-or-one inflated beta regression models', Computational Statistics and Data Analysis 56(6), 1609-1623.

Owen, C. (2008), Parameter estimation for the beta distribution, Master's thesis, Brigham young university.

Comunicaciones en Estadística, junio 2018, Vol. 11, No. 1 
Pace, L. \& Salvan, A. (1997), 'Principles of statistical inference from a neo-fisherian perspective. in: Advanced series on statistical science and applied probability', World Scientific 4, 128.

Qiu, Z., Song, P. \& Tan, M. (2008), 'Simplex mixed-effects models for longitudinal proportional data', Scandinavian Journal of Statistics 35(4), 577-596.

R Core Team (2017), R: A Language and Environment for Statistical Computing, $\mathrm{R}$ Foundation for Statistical Computing, Vienna, Austria. *http://www.R-project.org/

Rigby, B. \& Stasinopoulos, M. (2005), 'Generalized additive models for location, scale and shape', Applied Statistical 54(3), 507-554.

Rigby, B. \& Stasinopoulos, M. (2008), 'Instructions on how to use the gamlss package in R', Computational statistics and Data analysis 2, 194-195.

Zeileis, A., Cribari-Neto, F. \& Grün, B. (2010), 'Beta regression in R', Journal of statistical software 34(2), 1-24.

Zhang, P., Qiu, Z. \& Shi, C. (2016), 'simplexreg: An R package for regression analysis of proportional data using the simplex distribution', Journal of Statistical Sftware 71(11). 